\title{
Hot Phonons in a Biased Two-Dimensional InGaAs Channel
}

\author{
V. Aninkevičius*, J. Liberis, A. Matulionis \\ AND I. MATUlionienĖ \\ Semiconductor Physics Institute, A. Goštauto 11, Vilnius 01108, Lithuania \\ Experimental dependence of microwave noise temperature on supplied \\ electric power is used to estimate hot-phonon number in a modulation-doped \\ $\mathrm{In}_{0.52} \mathrm{Al}_{0.48} \mathrm{As} / \mathrm{In}_{0.53} \mathrm{Ga}_{0.47} \mathrm{As} / \mathrm{In}_{0.7} \mathrm{Ga}_{0.3} \mathrm{As} / \mathrm{In}_{0.52} \mathrm{Al}_{0.48} \mathrm{As}$ two-dimensional \\ electron gas channel $\left(n_{2 \mathrm{D}}=2.3 \times 10^{12} \mathrm{~cm}^{-2}\right)$. The nonequilibrium occupancy \\ of the involved longitudinal optical phonon states exceeds the equilibrium \\ one nearly twice at $2 \mathrm{kV} / \mathrm{cm}$ electric field.
}

PACS numbers: 63.20.Kr, 72.20.Ht

\section{Introduction}

Two-dimensional electron gas (2DEG) channels are widely used in high electron mobility transistors [1]. In a selectively doped InAlAs/InGaAs/InAlAs/InP heterostructure, the 2DEG forms in an undoped InGaAs layer, and the mobile electrons are supplied by a modulation-doped InAlAs layer. An In-rich InGaAs pseudomorphic layer forms a deep quantum well, and contains a high-density high-mobility 2DEG. These properties are useful for ultrafast operation, supposing that electron energy dissipation is efficient enough to keep the hot electrons confined in the quantum well. The main dissipation mechanism is emission of longitudinal optical (LO) phonons by high-energy electrons. The accumulated LO phonons, termed hot phonons [2], change electron energy dissipation. This has been evidenced for nitride 2DEG channels [3]. A similar study has not been carried out for arsenides, and the paper aims at estimating the effective LO-phonon number in the biased 2DEG channel located in a pseudomorphic $\operatorname{In}_{0.7} \mathrm{Ga}_{0.3}$ As layer of an InP-based heterostructure.

\section{Dependence of noise temperature on supplied power}

Squares in Fig. 1 present dependence of noise temperature on supplied electric power for $\mathrm{In}_{0.52} \mathrm{Al}_{0.48} \mathrm{As} / \mathrm{In}_{0.53} \mathrm{Ga}_{0.47} \mathrm{As} / \mathrm{In}_{0.7} \mathrm{Ga}_{0.3} \mathrm{As} / \mathrm{In}_{0.52} \mathrm{Al}_{0.48} \mathrm{As}$. The

*corresponding author; e-mail: aninkevicius@pfi.lt 


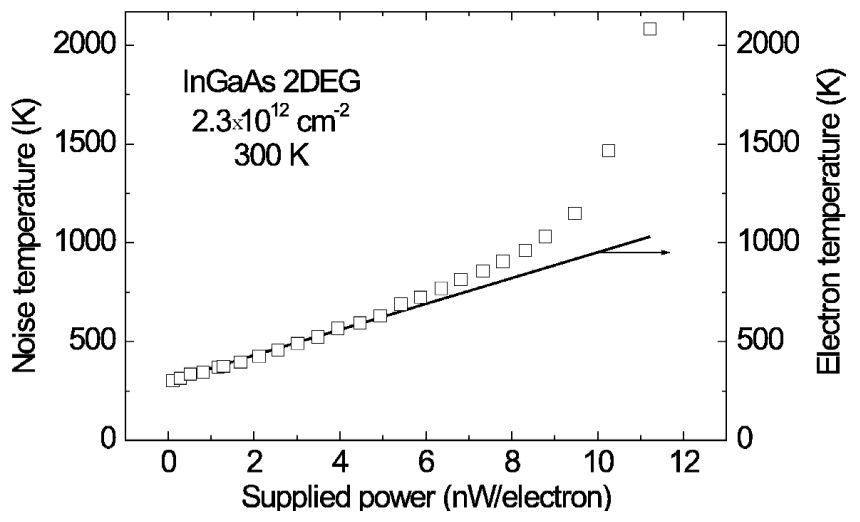

Fig. 1. Dependence on supplied electric power of measured longitudinal noise temperature (squares) and the electron temperature estimated after Eq. (2) (solid line) at $300 \mathrm{~K}$.

heterostructure consists of an undoped $200 \mathrm{~nm}$ lattice-matched $\operatorname{In}_{0.52} \mathrm{Al}_{0.48} \mathrm{As}$ buffer layer, an undoped lattice-matched $30 \mathrm{~nm}$ layer of $\operatorname{In}_{0.53} \mathrm{Ga}_{0.47} \mathrm{As}$, an undoped strained $12 \mathrm{~nm}$ layer of $\operatorname{In}_{0.7} \mathrm{Ga}_{0.3} \mathrm{As}$, and a $35 \mathrm{~nm} \mathrm{In}_{0.52} \mathrm{Al}_{0.48}$ As top layer. The Si-donor plane is located in the top InAlAs layer at $3 \mathrm{~nm}$ distance from the 2DEG channel formed in the strained layer. The electron sheet density is $n_{2 \mathrm{D}}=2.3 \times 10^{12} \mathrm{~cm}^{-2}$, and the mobility is $\mu_{0}=11200 \mathrm{~cm}^{2} /(\mathrm{V} \mathrm{s})$ at room temperature.

The supplied electric power per electron $P_{\mathrm{s}}$ is

$$
P_{\mathrm{s}}=U I / N_{\mathrm{e}}
$$

where $U$ is the voltage, $I$ is the current, $N_{\mathrm{e}}$ is the electron number in the channel.

Since the 2DEG density is high, the electron temperature can be introduced. It depends on the supplied power $P_{\mathrm{s}}$ :

$$
T_{\mathrm{e}}=T_{0}+P_{\mathrm{s}} \tau_{\varepsilon} / k_{\mathrm{B}}
$$

were $\tau_{\varepsilon}$ is the electron energy relaxation time, $T_{0}$ is the ambient temperature, and $k_{\mathrm{B}}$ is the Boltzmann constant.

The electron temperature was estimated from noise temperature measurements at a microwave frequency near $10 \mathrm{GHz}$, where generation-recombination, flicker, and other low-frequency sources of noise are of negligible intensity $[4,5]$.

Solid line in Fig. 1 illustrates Eq. (2) for $\tau_{\varepsilon}=0.9 \mathrm{ps}$; this value is taken from Ref. [4]. At a low supplied power, the noise temperature equals the electron temperature.

\section{Power dissipation on equilibrium LO phonons}

A six-subband model is used to discuss the experimental results. The electron envelope wave functions and eigenenergies for the lowest subbands are ob- 
tained through self-consistent solution of the coupled Schrödinger and Poisson equations. The required subband occupancy is determined by the Fermi-Dirac distribution function multiplied with the density of states in the corresponding subband and integrated over the electron energy.

Interaction of hot electrons with LO phonons is the main electron energy relaxation mechanism in InGaAs 2DEG channel at room temperature [6]. In general, different LO-phonon modes are of importance in the InGaAs 2DEG channel: confined InAs-like phonons coexist with GaAs-like phonons and interface phonons. The joint effect due to the confined and interface modes can be quite accurately represented with bulk-like phonons [7]. For the very beginning, let us discuss the experimental data on hot-electron energy dissipation in terms of equilibrium dispersionless bulk-like LO phonons.

In the assumed approach, the equilibrium LO-phonon number $N_{\mathrm{ph}}^{\mathrm{eq}}$ is determined by the absolute temperature $T$ according to the Bose-Einstein distribution function

$$
N_{\mathrm{ph}}^{\mathrm{eq}}=\left[\exp \left(\frac{\hbar \omega}{k_{\mathrm{B}} T}\right)-1\right]^{-1},
$$

where $\hbar \omega$ is the LO-phonon energy.

The dissipated power per electron is treated in terms of two effective time constants: one for spontaneous emission of an LO phonon by a high-energy electron, $\tau_{\mathrm{sp}}$, and another one for a phonon absorption, $\tau_{\mathrm{ab}}$. The dissipation rate $P_{\mathrm{d}}$ is

$$
P_{\mathrm{d}}=\hbar \omega\left(\frac{1+N_{\mathrm{ph}}^{\mathrm{eq}}}{\tau_{\mathrm{sp}}} p_{-}-\frac{N_{\mathrm{ph}}^{\mathrm{eq}}}{\tau_{\mathrm{ab}}} p_{+}\right),
$$

where $1+N_{\mathrm{ph}}^{\mathrm{eq}}$ takes into account spontaneous and stimulated emission of LO phonons, the last term takes into account LO phonon absorption, $p_{ \pm}$are the probabilities to find an electron able to emit $(-)$ or absorb $(+)$ an LO phonon, respectively. The time for spontaneous emission was assumed independent of the electron energy. The values for $\tau_{\mathrm{ab}}$ can be obtained from condition $P_{\mathrm{d}}=0$ that corresponds to the thermal equilibrium.

The probabilities to find proper electrons are

$$
p_{ \pm}=\frac{\int_{0}^{\infty} D(\varepsilon) f(\varepsilon) \Omega_{ \pm}[1-f(\varepsilon \pm \hbar \omega)] \mathrm{d} \varepsilon}{\int_{0}^{\infty} D(\varepsilon) f(\varepsilon) \mathrm{d} \varepsilon},
$$

where $\varepsilon$ is the electron energy, $D(\varepsilon)$ is the density-of-state function (Fig. 2, solid line), $\Omega_{ \pm}$is the unity step function $\left(\Omega_{-}=0\right.$ at $\varepsilon<\hbar \omega, \Omega_{-}=1$ at $\varepsilon>\hbar \omega$, and $\left.\Omega_{+}=1\right), f(\varepsilon)$ is the hot-electron energy distribution function. The factor $1-f$ takes into account the electron gas degeneracy.

Dashed and dotted lines in Fig. 2 illustrate the integrands of the numerators in Eq. (5) calculated in the electron-temperature approximation with the FermiDirac distribution taken into account. The spontaneous emission time and the 


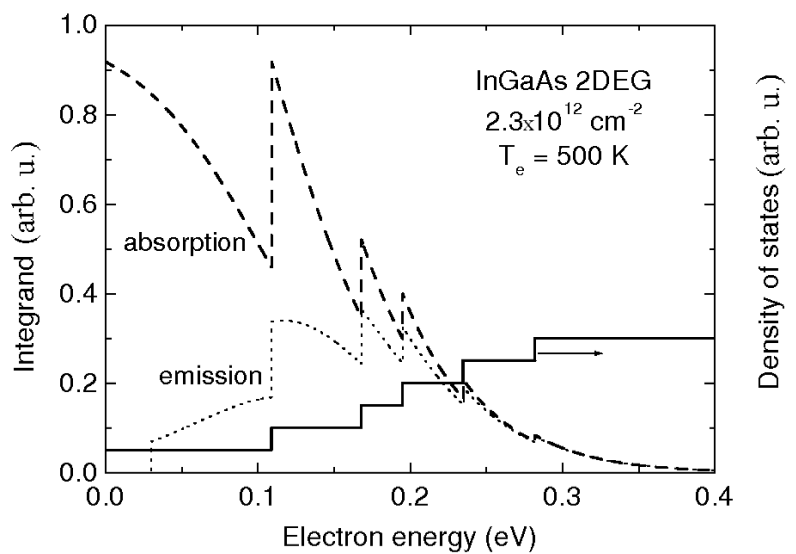

Fig. 2. Dependence on electron energy of density-of-states (solid line) and integrands of numerators in Eq. (5) for emission (dots) and absorption (dashes) at $T_{\mathrm{e}}=500 \mathrm{~K}$. LO-phonon energy $\hbar \omega=0.03 \mathrm{eV}$.

LO-phonon lifetime are not known for the heterostructure in question; the chosen values, $\tau_{\mathrm{sp}}=160$ fs and $\hbar \omega=0.03 \mathrm{eV}$, are close to (in between) those for GaAs and InAs $[8,9]$.

Figure 3 compares the measured supplied power (squares) with the calculated dissipated power. The latter depends on the electron temperature according to Eq. (4); solid line presents the result for $\tau_{\mathrm{sp}}=160 \mathrm{fs}$ and $\hbar \omega=0.03 \mathrm{eV}$. In the range $P_{\mathrm{s}}<5 \mathrm{nW}$, the same scale can be used for the noise temperature and the electron temperature. The comparison shows that the calculated dependence is similar to the measured. The misfit would be even stronger if one took into account more dissipation mechanisms in addition to the electron-LO-phonon interaction.

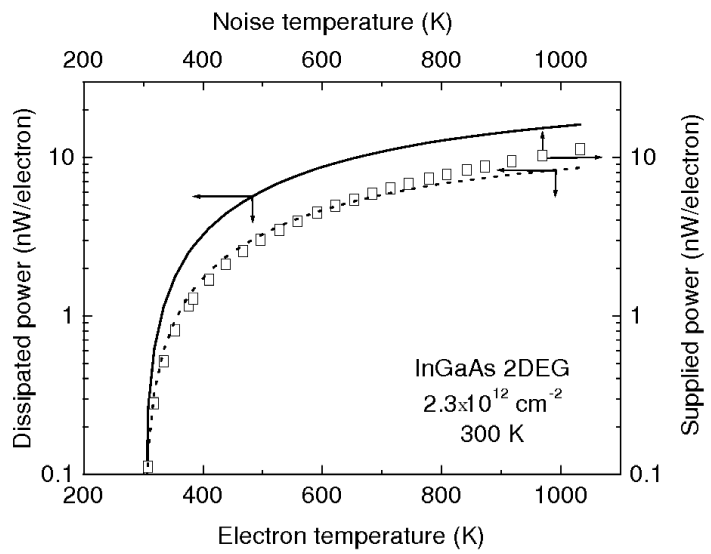

Fig. 3. Experimental results on supplied power (squares) and dissipated power (lines) calculated after Eq. (4) at $300 \mathrm{~K}$ for two values of spontaneous emission time $\tau_{\mathrm{sp}}: 160 \mathrm{fs}$ (solid line) and $300 \mathrm{fs}$ (dotted line). 
A satisfactory fitting could be obtained if one assumed $\tau_{\mathrm{sp}}=300 \mathrm{fs}$ (dotted line in Fig. 3). However, if this value was accepted for the spontaneous emission time, the electron mobility values would be overestimated. Consequently, no acceptable solution was obtained when only equilibrium phonons were taken into account.

\section{Power dissipation on hot phonons}

The dissipated power is lower if the equilibrium phonon number in Eq. (4) is replaced with an effective nonequilibrium phonon number $\tilde{N}_{\mathrm{ph}}$ that exceeds $N_{\mathrm{ph}}^{\mathrm{eq}}$.

Under the steady state the supplied power is balanced with the dissipated power; Eqs. (1) and (4) lead to

$$
\tilde{N}_{\mathrm{ph}}=\frac{\frac{p_{-}}{\tau_{\mathrm{sp}}}-\frac{U I / N_{\mathrm{e}}}{\hbar \omega}}{\frac{p_{+}}{\tau_{\mathrm{ab}}}-\frac{p_{-}}{\tau_{\mathrm{sp}}}} .
$$

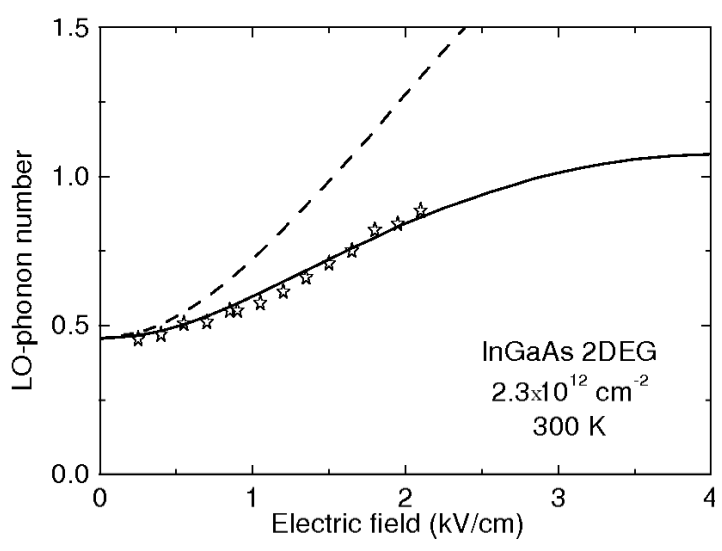

Fig. 4. Dependence on electric field of the effective hot-phonon number estimated according to Eq. (6) where $p_{ \pm}$is calculated after Eq. (5): stars correspond to $T_{\mathrm{e}}=T_{\mathrm{n}}$ (squares in Fig. 1); solid line is obtained for $T_{\mathrm{e}}$ approximation (solid line, Fig. 1). Dashed line is Eq. (3) where $T=T_{\mathrm{e}}$. Spontaneous emission time $\tau_{\mathrm{sp}}=160 \mathrm{fs}$.

Figure 4 presents the results obtained according to Eq. (6). Stars (Fig. 4) are obtained when the measured noise temperature as a function of the supplied power is used in the range where $T_{\mathrm{n}} \approx T_{\mathrm{e}}$ (squares, Fig. 1). Solid line (Fig. 4) uses $T_{\mathrm{e}}\left(P_{\mathrm{s}}\right)$ approximation (solid line, Fig. 1). The results are compared with $N_{\mathrm{ph}}\left(T_{\mathrm{e}}\right)$ (dashed line): the quantity $N_{\mathrm{ph}}\left(T_{\mathrm{e}}\right)$ is obtained according to Eq. (3) where $T_{\mathrm{e}}$ is substituted for $T$.

In conclusion, the experimental data on supplied electric power and microwave noise temperature are used for estimation of the nonequilibrium LO-phonon number in the biased InP-based InGaAs two-dimensional channel. The nonequilibrium occupancy of the involved LO-phonon states exceeds the equilibrium one nearly twice at $2 \mathrm{kV} / \mathrm{cm}$ electric field. 


\section{Acknowledgments}

Support provided under grant No. N00014-03-1-0558 of the USA Office of Naval Research is acknowledged.

\section{References}

[1] S. Tiwari, Compound Semiconductor Device Physics, Academic Press, New York 2002.

[2] P. Kocevar, Physica $B+C$ 134, 155 (1985).

[3] A. Matulionis, J. Liberis, I. Matulionienè, M. Ramonas, L.F. Eastman, J.R. Shealy, V. Tilak, A. Vertiatchikh, Phys. Rev. B 68, 035338 (2003).

[4] A. Matulionis, V. Aninkevičius, J. Liberis, I. Matulionienė, J. Berntgen, K. Heime, H.L. Hartnagel, Appl. Phys. Lett. 74, 1895 (1999).

[5] H.L. Hartnagel, R. Katilius, A. Matulionis, Microwave Noise in Semiconductor Devices, Wiley, New York 2001.

[6] L. Ardaravičius, J. Liberis, A. Matulionis, M. Ramonas, Fluctuation and Noise Letters 2, L53 (2002).

[7] P. Bordone, P. Lugli, Phys. Rev. B 49, 8178 (1994).

[8] K.T. Tsen, H. Morkoç, Phys. Rev. B 38, 5615 (1988).

[9] E. Kobayashi, C. Hamaguchi, T. Matsuoka, K. Taniguchi, IEEE Trans. Electron Dev. 36, 2353 (1989) 